\title{
Practice on Meter Dose Inhaler Techniques and Its Associated Factors Among Asthmatic Patients at Debre Markos Compre- hensive Specialized Hospital, East Gojjam, Ethiopia: A Pro- spective Study.
}

\author{
Tadele Asmare, MD ${ }^{1}$, Anteneh Belayneh, MSc'2, Bekalu Dessie , $\mathrm{MSc}^{2 *}$
}

${ }^{1}$ School of Medicine, Debre Markos University, Debre Markos, Ethiopia

2 Department of Pharmacy, College of Health Sciences, Debre Markos University, Debre Markos, Ethiopia

* Correspondence: Bekalu Dessie (B. pharm, MSc in clinical pharmacy), Department of Pharmacy, College of Health Sciences, Debre Markos University, P.O. Box 269, Ethiopia; Email; bekiebda@gmail.com $\stackrel{\text { Phone No: }}{ }$ $+251913866884$

\begin{abstract}
Background: Asthma is ranked $16^{\text {th }}$ among the leading causes of years lived with disability and $28^{\text {th }}$ among the leading causes of disease in the world. Metered dose inhaler remains the principal route for drug administration and it has greater advantages over systemic treatment. In routine use, however, a vast majority of patients make inhalation errors. Suboptimal inhaler technique worsens health outcomes, with poor disease control, and increases the risk of hospitalization. This study aimed to assess practice metered-dose inhalation techniques and associated factors among asthmatic patients at Debre Markos comprehensive specialized hospital, East Gojjam, Amhara region, Ethiopia. Methods: Prospective cross-sectional study was conducted from July 15 to August 30/ 2020. Data were collected through a semi-structured questioner. The data was analyzed using SPSS version 25. Associations between dependent and independent variables were assessed by using binary and multiple logistic regressions. P-values less than 0.05 were considered to be statistically significant in all cases. Results presented in tables, figures, numbers, and percentages. Result: A total of 166 patients had involved in the study, of which $52.4 \%$ were females. One hundred eleven $(66.9 \%)$ of participants had good knowledge of asthma and inhalational techniques with the rest of them had poor knowledge. One hundred eight (65.1\%) patients had effective practice on metered-dose inhaler use techniques. Participant's knowledge regarding asthma and meter dose inhaler and marital status has a significant association with their practice of metered-dose inhaler techniques with P-value 0.001 and 0.006 respectively. Conclusion: In this study, most participants are suffering from asthma for a long duration and they have repeated exacerbation. Around twothirds of patients had good knowledge regarding asthma and meter dose inhaler and practice on metered-dose inhaler techniques. Participants who had poor knowledge and who were unmarried had poor practice on tech metered-dose inhaler techniques. Health education and counseling services should be consistently provided to the clients regarding the proper steps of inhalers use.
\end{abstract}

Keywords: Asthma; meter dose inhaler; Debre Markos comprehensive specialized hospital; Practice

\section{Introduction}

Asthma is a persistent inflammatory disease of the airways in which many cells and cellular elements play a role which leads to recurrent episodes of wheezing, breathlessness, chest tightness and coughing. ${ }^{1}$ Asthma is ranked $16^{\text {th }}$ among the leading causes of years lived with disease and $28^{\text {th }}$ among the leading causes of disease in the world. The disease affects $1-18 \%$ population in the world. ${ }^{2}$

Asthma continues to a global economic burden. In the USA, the total asthma costs increased from 53 billion US dollars in 2002 to 56 billion US dollars in 2011. Accurate estimates of costs of treatments are not available for majority of developing countries. Better disease management, improving access to health care, especially to preventer therapies \& 
improved adherence to such therapies significantly reduce the financial burden of the disease. Above $80 \%$ of asthma mortalities occur in developing countries ${ }^{3-5}$

Inhalation therapy forms are the mainstay of the treatment of asthma. Inhalation medications have fewer systemic side effects and immediate action than oral asthma medication. However, they need a skill that $70-80 \%$ of patients are unable to use inhaler correct Meter dose inhaler (MDI) remains the principal route for drug administration and it has greater advantages over systemic treatment. In routine use, however, a vast majority of patients make inhalation errors. Suboptimal inhaler technique worsens health outcomes, with poor disease control, and increases the risk of hospitalization. So, this study aims to know the cause of suboptimal usage and to assess the correct usage of MDI finally it will improve the care and treatment of an asthmatic patient.1, 4, 6-8

A cross-sectional study done at New Delhi, India on 300 patients showed that $86 \%$ patients identified their disease as breathlessness and $65 \%$ of them described it as repeated attacks of cough with or without expectoration. ${ }^{8}$ In another cross-sectional survey conduct in Abu Dhabi, $67.7 \%$ parents were confident about the use of inhalers. Almost half (50.4\%) of parents were aware of an asthma action plan, and $42.5 \%$ knew the correct storage method for inhalers. ${ }^{9}$

A descriptive study conducted among 105 patients in public hospitals in Sudan to assess knowledge and practice of asthmatic patients regarding the use of MDIs reflected the knowledge score of participants about steps of inhalation dose, steps of care post inhalation, storage and clean the device respectively is $77 \%, 44 \%, 79 \%, 43 \%$, while participants had a poor level of knowledge about preparation of the dose, replacement and cleaning mouthpiece with respective proportions of $63 \%, 68 \%, 60 \% .^{10}$

A case-control study was conducted to evaluate determinants of uncontrolled asthma in Jimma university medical center on 121 controls and 121 cases showed that $112(92.6 \%)$ of the controls and $35(28.9 \%)$ of the cases had good knowledge about asthma. ${ }^{11}$ In another study done at Sudan, the level of participant's knowledge was very good regard the care and storage of the device.$^{12}$ Most asthmatic patients can't use their inhaler correctly. At least 50 \%adults and children didn't take controller medication as prescribed this will cause poor control of symptoms..$^{13}$

A prospective interventional study on knowledge, attitude and practice towards MDI techniques conducted in India showed among 50 participants $86.1 \%$ were using inhalers incorrectly. The common mistakes encountered on $g$ inhaler techniques were: not shaking the canister before use, unable to breathe hold, exhalation through the mouth, not washing the mouth after using inhalers. In similar study, 33\% of the patients were not convinced about diagnosis of asthma and many had poor knowledge about inhalant therapy. ${ }^{14}$

In a study on 190 asthmatics having a follow up at department of pulmonary medicine done at Patiala college of health science showed about $74 \%$ preferred use of inhalers for asthma. However, about $43 \%$ liked to use oral medications more than inhaled medicines. About $55 \%$ believed that inhaled medications contain a higher dose than oral (7).

An observational study done in India on an incorrect inhaler technique showed that, out of 89 patients using MDI, only 10(11.2\%) patients could demonstrate all the steps of inhaler usage correctly. $88.7 \%$ of patients had errors in one or more steps of the inhaler technique. The most common error was not holding the breath for 10 seconds which was seen in $46(51.6 \%)$ patients. Thirty $(33.7 \%)$ patients had an error of failure to exhale residual volume before inhalation. Three (3.3\%) patients did not remove the cap from the canister and were unable to hold the inhaler upright.$^{15}$

The result of a research done at different hospitals in Abu Dhabi also showed the attitude of patients towards asthma and their behavior regarding the use of inhalers and preventive therapy, $57 \%$ of patients usually seek medical care for treatment of asthma. $59 \%$ of asthma patients did not use anti-inflammatory drugs in the form of steroid inhalers when prescribed because they were afraid of lifelong dependence on inhalers. Most (76\%) 
patients have felt safe taking their drugs when they were at work or away from home or outside of home. ${ }^{16}$

A Prospective study conducted among 140 patients on MDI use technique at Jima University specialized hospital showed that most (92.85\%) patients had errors in one or more steps of inhaler technique. The commonest error was found to not inhaling slowly, simultaneously not pressing the canister, and failing to breath in slowly and deeply which was committed by $69.28 \%$ of patients. The next frequent error was lean head slightly back, $49.3 \%$, and take the inhaler out of the mouth and hold the breath for 5-10 sec was the third most missed step which accounts for $38.6 \% .{ }^{17}$

There are limited research findings to determine knowledge, and practice towards inhalation techniques among asthmatic patients in Ethiopia and specifically, there is no study at comprehensive specialized hospital (DMCSH). There is repeated exacerbation of asthma in patients who have followed up at DMCSH who are on treatment. This study is, therefore, directed to investigate the level of practice MDI and associated factors among patients have follow-up DMCSH.

\section{Methodology}

\section{Study Area and Study Period}

This study was conducted at DMCSH which is found in Debre Markos town. DMCSH has 4 major and 3 minor departments; medicine, surgery, pediatrics, GYN-obs, ophthalmology, psychiatry, and derma clinic. The medical department has also, ward, emergency, medical referral clinic, and 3 cold outpatient department (OPD), emergency, and medical referral clinic. ${ }^{18}$

The study was conducted from July 15, 2020to August 30, 2020.

\section{Study Design}

A hospital-based cross-sectional study was conducted to evaluate practice towards metered-dose inhaler technique among asthmatic patients who visited DMCSH during the study period (from July 15, 2020 to August 30, 2020.

\section{Source Population}

The source populations for this study were all asthmatic patients who have follow up visit at DMCSH.

\section{Study Population}

The source populations for this study were all asthmatic patients who have followup visits at DMCSH who came during the study period.

\section{Inclusion Criteria}

Patients with known cases of asthma, with age above 18 years and took inhalational medicine for at least 03 months, who were willing to take part in the study were enrolled for the study.

\section{Exclusion Criteria}

All asthmatic patients who are seriously ill during the data collection period and had other co-morbid medical conditions were excluded from the study.

\section{Sampling Technique and Sample Size}

The sampling technique was convenient sampling. All cases who visited the hospital during the study period based on inclusion criteria were included in the study. The total sample size of the study was 166 participants. 
Data was collected by using semi-structured questionnaires prepared by adapting from different literatures. ${ }^{7,11,14}$ The questionnaire has three parts; socio-demographic characteristics, knowledge \& practices of asthmatic patients towards the use of meter dose inhalers. The Data was collected by the data collectors from the study participants. Demographic data were collected at the time of the interview, which includes age, gender, education status, occupation, area of residence, frequency of inhaler use. A semi-structured interview schedule regarding the use of inhalers is administered to collect the data. The interview questions were designed based on previous studies on this subject to assess the practice of the patients towards inhaler use. At the end of the interview, participants were allowed to share any of their concerns or opinions regarding inhaler use other than those mentioned in the proforma. Afterward, the interviewers spend time with the patient to educate them regarding any of their ill-knowledge or misconceptions about inhalation therapy.

\section{Data Analysis}

Data entry, coding, and analysis were performed using SPSS version 25 software package. frequencies, percent, and summary statics were used to describe variables. Associations between dependent and independent variables was assessed by using binary and multiple logistic regressions. To measure internal consistency, reliability analysis was done. p-values less than 0.05 were considered to be statistically significant in all cases.

\section{Operational Definition} tions,

Good knowledge; those respondents who answer 50\% and above of knowledge ques-

Poor knowledge; those respondents who answer less than $50 \%$ of knowledge questions, there are 8 questions on the inhalation technique were identified using a standard checklist of steps recommended by NIH guideline with one point given for each inhalational technique performed correctly with a maximum of a score being 8 , four or more correct response is labeled as efficient and if the correct steps are less than 4 were assigned as inefficient. ${ }^{14}$

\section{Dependent Variable}

Respondent's practice on MDI was the dependent variable

\section{Independent Variables}

Respondent's sociodemographic variable; age, sex, educational status, religion, residency duration of asthma, knowledge towards MDI use were the independent variable of the study.

\section{Result}

\section{Sociodemographic Characteristics}

A total of 166 patients participated in the study, of which 87 (52.4\%) were females and $79(47.6 \%)$ were males. The average age of the participants was 41.10 years with a minimum age of 20 and a maximum age of 80 years. About $85(51.2 \%)$ are residing in an urban area (Table1).

Table 1. Sociodemographic characteristics of the respondents DMCSH, 2020.

\begin{tabular}{|c|c|c|c|c|}
\hline & & \multicolumn{3}{|c|}{ Sex } \\
\hline & & MALE & Female & Total \\
\hline \multirow{2}{*}{ Residency } & Urban & 36 & 49 & 85 \\
\hline & Rural & 43 & 38 & 81 \\
\hline \multirow{2}{*}{ Age } & $18-40$ & 32 & 47 & 79 \\
\hline & $40-65$ & 44 & 35 & 79 \\
\hline
\end{tabular}




\begin{tabular}{|c|c|c|c|c|}
\hline & $>=65$ & 3 & 5 & 8 \\
\hline \multirow{5}{*}{ Level of Education } & Unable to Read and Write & 32 & 22 & 54 \\
\hline & Primary School & 18 & 14 & 32 \\
\hline & Secondary School & 12 & 17 & 29 \\
\hline & Diploma and Above & 17 & 34 & 51 \\
\hline & Total & 79 & 87 & 166 \\
\hline \multirow{4}{*}{ Occupation } & Government & 9 & 19 & 28 \\
\hline & Private & 21 & 44 & 65 \\
\hline & Others & 49 & 24 & 73 \\
\hline & Total & 79 & 87 & 166 \\
\hline \multirow{5}{*}{ Marital Status } & Single & 18 & 1 & 19 \\
\hline & Married & 49 & 44 & 93 \\
\hline & Divorced & 1 & 6 & 7 \\
\hline & Widowed & 11 & 36 & 47 \\
\hline & Total & 79 & 87 & 166 \\
\hline
\end{tabular}

\section{Participants' Clinical Condition}

From a total of 166 participants, the minimum and maximum duration of asthma were 7 and 70 years respectively with a mean duration of 27 years. Fourteen $(8.4 \%)$ patients were suffering from asthma for a duration of less than or equal to ten years and $76(45.8 \%)$ are suffering from asthma for more than twenty-seven years with a maximum of 70 - years (table 2 ).

Table 2. Duration of asthma of patients at DMCSH, 2020.

\begin{tabular}{ccccc}
\hline & \multicolumn{3}{c}{ Sex } & \\
\cline { 2 - 3 } Duration of asthma & Male & Female & N $(\%)$ \\
\hline$<=10$ years & 5 & 9 & $14(8.4)$ \\
\hline $10-27$ year & 32 & 44 & $76(45.8)$ \\
\hline$>27$ year & 42 & 34 & $76(45.8)$ \\
\hline Total & 79 & 87 & $166(100)$ \\
\hline
\end{tabular}

Knowledge about the meter dose inhaler asthmatic patient

All participants were aware of their diagnosis. The commonest side effect was fast heart beat which has been reported by 119 (71.6\%) patients while 36(21.7\%) patients were complaining of shaking of hands. One hundred fifty-six (94\%) of respondents used relievers in the time of acute exacerbation of asthma. One hundred eleven $(66.9 \%)$ of participants had good knowledge,55(33.1\%) participants had poor knowledge regarding asthma and MDI (Figure 1).

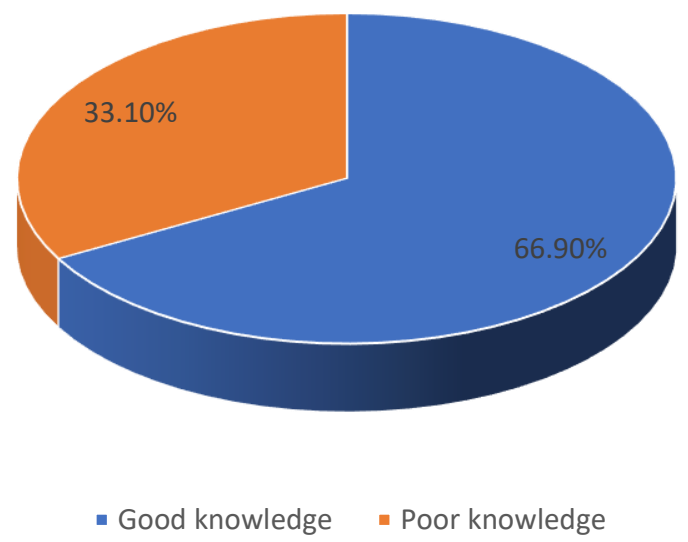

Figure 1. Knowledge regarding the disease and meter dose inhaler, DMCSH, 2020. 
Practice of Participant on MDI use

All of the respondents believe that they can use the inhaler correctly. All participants were not using refrigerator as storage. Most of them store their inhaler at room temperature away from direct sunshine exposure (Table 3).

Of 166 respondents, $108(65.1 \%)$ patients were using the inhaler effectively and the remaining $58(34.9 \%)$ used the inhaler device ineffectively. The commonest mistake in using the inhaler was failing to check the label and expiry date (74\%), not holding breath for 10 seconds $(67.5 \%)$, and not breathing out gently (66.3\%). Most participants practiced shaking well $(79.5 \%)$ and replacing cap $(72.3 \%)$ steps (Table 3$)$.

Table 3. Practice of inhaler uses within the respondents, DMCSH, 2020.

\begin{tabular}{lccc}
\hline \multirow{2}{*}{ No } & Questions & YES & NO \\
\cline { 2 - 4 } & Check the expire date & N (\%) & N (\%) \\
\hline 1 & Hold upright and shake well before use & $43(25.9)$ & $123(74.1)$ \\
\hline 2 & Breath out gently & $132(79.5)$ & $34(20.5)$ \\
\hline 3 & form good seal & $56(33.7)$ & $110(66.3)$ \\
\hline 4 & Put the mouthpiece between the teeth without biting and closed lips to & $158(95.2)$ & $8(4.8)$ \\
\hline & Start to breath in slowly through mouth and press down firmly on canister & & \\
\hline & and continue to breath in slowly and deep & 144(86.7) & $22(13.3)$ \\
\hline 6 & Hold breath for 10 seconds then remove inhaler from the mouth & $54(32.5)$ & $112(67.5)$ \\
\hline 7 & Breath out gently & $96(57.8)$ & $70(42.2)$ \\
\hline 8 & Replace the cap & $120(72.3)$ & $46(27.7)$ \\
\hline
\end{tabular}

Factors associated with practices towards MDI

In this study, level of knowledge towards asthma and its management, residence, level of education, marital status and occupation showed association with poor practice on bivariable logistic regression. But only knowledge and marital status showed a significant association on multivariable logistic regression. Patients with more knowledge were 12 times more likely to have poor practice on MDI device use compared to those who had good knowledge (AOR=12, 95\%CI: 4.5,31.9). Single patients were 0.8 times less likely to have poor practice on MDI compared to those who were widowed (AOR=0.196, 95\%CI: 0.05-0.8)(Table 4). 
Table 4. Factors associated with practices towards MDI use in DMCSH, 2020.

\begin{tabular}{|c|c|c|c|c|c|c|}
\hline \multirow{2}{*}{ Variables } & \multicolumn{2}{|c|}{ PRACTICE } & \multirow{2}{*}{ COR, 95\%CI } & \multirow{2}{*}{$\mathrm{p}$-Value } & \multirow{2}{*}{$\mathrm{AOR}, 95 \% \mathrm{CI}$} & \multirow{2}{*}{$\mathrm{p}$-Value } \\
\hline & Good & Poor & & & & \\
\hline \multicolumn{7}{|c|}{ Sex } \\
\hline Male & 51 & 28 & 1.00 & & & \\
\hline Female & 57 & 30 & $.959(.506,1.816)$ & .897 & & \\
\hline \multicolumn{7}{|c|}{ Marital Status } \\
\hline Single & 13 & 6 & $0.4(0.13-1.2)$ & 0.116 & $0.196(0.05-0.8)$ & 0.012 \\
\hline Married & 66 & 27 & $0.36(0.174-0.74)$ & 0.006 & 0.3 & 0.45 \\
\hline Divorced & 7 & 0 & 0.000 & 0.99 & 0.00 & 0.00 \\
\hline Widowed & 22 & 25 & 1 & & & \\
\hline \multicolumn{7}{|c|}{ Occupation } \\
\hline Government & 26 & 2 & 1 & & & \\
\hline Private & 36 & 29 & $0.01(0.694-2.7)$ & 0.009 & 1.9 & 0.5 \\
\hline Others & 46 & 27 & $0.13(0.03-0.6)$ & 0.01 & 1.75 & \\
\hline \multicolumn{7}{|c|}{ Residency } \\
\hline Urban & 65 & 20 & 1.00 & & 1.00 & \\
\hline Rural & 43 & 38 & $0.35(0.18-0.7)$ & 0.002 & $2.4(0.7-7.8)$ & 0.14 \\
\hline \multicolumn{7}{|c|}{ Knowledge } \\
\hline Good Knowledge & 91 & 20 & 1.00 & & 1.00 & \\
\hline Poor Knowledge & 17 & 38 & $10(4.8,21.5)$ & $<0.001$ & $12(4.5,31.9)$ & $<0.001$ \\
\hline
\end{tabular}

\section{Discussion}

Numerous studies on knowledge and practices of patients regarding their illness are potent contributing factors to disease management. Asthma is a chronic disease in which a patient's good behaviors and practices are more significant in controlling the disease. The finding of this study revealed that 111 (66.9\%) participants had good knowledge and $55(33.1 \%)$ had poor knowledge on asthma and inhalational techniques, however a casecontrol study done at Jimma university medical center reported $35(28.9 \%)$ patients had good knowledge and $86(71.5 \%)$ patients on the case had also poor knowledge. ${ }^{11}$ This discrepancy might be due to the socio-demographic dissimilarities between the two populations i.e. in our study more than two-thirds of the patients are attending secondary school but in the study done at Jimma, more than half of the participants were illiterate. In another study done at Jimma $61.5 \%$ were efficient in using inhaler which is in line with our finding CI 95\% (57-71\%). ${ }^{17}$

In our study, most of the patients believe that they can use the inhaler correctly, but only $108(65.1 \%)$ patients use the inhaler effectively and the remaining 58(34.9\%) were using the inhaler ineffectively. The commonest mistake encountered was failing to check labels and expire dates followed by not withholding breath for 10 seconds. Only 20(12\%) participants were performing all steps correctly. In the study done in India, from 200 participants showed, only $28(14 \%)$ patients performed all steps correctly in use of inhaler devices and 172(86\%) patients were unable to use inhalers properly. The most common error for MDIs was being unable to hold breathe for 10 seconds which was seen in $46(51.7 \%)$ patients. This difference could be explained by the socio-demographic and setups of the studies.15

On multivariable regression, only marital status and level of knowledge had a significant association Being married is preventive of married is less likely to have poor MDI use practice. Participants who had poor knowledge 12 times more likely to have poor MDI use practice than those with good knowledge. The study done at Jimma there showed a significant association between knowledge and practice. ${ }^{11}$ 
There was a small number of sample size which is due short period of data collection. The study sampling method was a nonprobability sampling method. so, is difficult to generalize the study for all asthmatic patients. The study was based on self-reported information which is exposed to self-report bias.

\section{Conclusion}

In this study, most participants are suffering from asthma for a long duration and they have repeated exacerbation. Around two-thirds of patients have good knowledge the rest have poor knowledge on asthma. More than half of the participants had a good practice on MDI. Most participants who had poor knowledge had poor practice. Health education and counseling services should be consistently provided to the clients regarding the proper steps of inhalers use.

Ethical Approval: The study was approved by the ethical review committee of the school of medicine, Debre Markos University. Oral consent was obtained from participants before being involved in the study. Confidentiality of data was assured for participants.

Acknowledgments: We are grateful for Debre Markos university for providing material support. Our sincere appreciation goes to also DMCSH staffs for their assistance during data collection.

\section{References}

1. GINA, global strategy of asthma management and prevention, Global Initiative for Asthma(GINA). 2014:56

2. World health organization(WHO), Global Asthma Report. 2018.

3. WHO. Global surveillance, prevention and control of chronic respiratory diseases: a comprehensive approach. 2007:21

4. Ait-Khaled N, Enarson DA, Bissell K, Billo NE. Access to inhaled corticosteroids is key to improving quality of care for asthma in developing countries. Allergy. 2007;62(3):230-6.

5. Thomas A, Lemanske RF, Jr., Jackson DJ. Approaches to stepping up and stepping down care in asthmatic patients. J Allergy Clin Immunol. 2011;128(5):915-26.

6. Wubaye walelign YF, Dejuma Yadeta,etal Ethiopian National Guidelines on Clinical and Programmatic Management of Major Noncommunicable Diseases, Addis Ababa. 2016.

7. Harjindersingh SPS, Jebin Abraham, Avneet kaur. A Study on Knowledge, Attitude and Practice of Asthmatic Patients towards Inhaler Use. IOSR Journal of Dental and Medical Sciences (IOSR-JDMS). 2019;8(4):06-10.

8. Rakhee Sodhi RP, R A S Kushwaha, Surya Kant et.al,. A study to know the knowledge, attitude, and practices of patients of bronchial asthma. 2019.

9. Lateefa Ahmed Al-Ali SAJ, Laila Mohammed Al Yammahi, Adnan Syeed, Ebtihal Ahmed Darwish Parental knowledge, attitudes, and practices regarding the use of prescribed inhalers in asthmatic children attending Ambulatory Healthcare Services Clinics,. Ibnosina Journal of Medicine and Biomedical Sciences April-June 2019, . 2019;11(2).

10. Mustafa HE, Rokza MSA, Elfak BA. Knowledge and Practice of Asthmatic's Patients Regard using Meter Dose Inhaler. IJLSSR by Society for Scientific Research under a CC BY-NC 40 International License 2018;4(6).

11. Ameha Zewudie TN, Yitagesu Mamo, Kabaye Kumela. Determinants of poorly controlled asthma among asthmatic patients in Jimma University Medical Center, Southwest Ethiopia, . BMC Research Notes. 2019;12:525.

12. Elbashir H, Rokza M, Elfaki B. Knowledge and Practice of Asthmatic's Patients Regard using Meter Dose Inhaler. International Journal of Life-Sciences Scientific Research. 2018;4:2080-7.

13. GINA, pocket guide for asthma management a nd prevention. 2019:27.

14. PadmavathyR, GeethuN, SajilaSylusS, ZachariaThomas, . assessment of knowledge, attitude, practice towards asthma and the impact of patient counselling on inhalation techniques in asthmatics. international research journal of pharmacy. 2019;10(6).

15. Sodhi MK. Incorrect inhaler techniques in western india International Journal of Research in Medical Sciences. 2017; 5(8):3461-5.

16. Osama Moheb Ibrahim Mohamed W. Knowledge, attitude and behaviour of asthmatic patients regarding asthma in primary care setting in Abu Dhabi. middle east journal of family medicine 2015;13(5).

17. Bezie Kebede, Grma Mamo, and Abebaw Molla. Association of Asthma Control and Metered-Dose Inhaler Use Technique among Adult Asthmatic Patients Attending Outpatient Clinic, in Resource-Limited Country: A Prospective Study. Hindawi Canadian Respiratory Journal 2019.

18. Debre Markos Comprehensive Specialized Hospital lie Zen office report, December 2020 